\title{
Racial and Ethnic Disparities in Discharge to Rehabilitation Following Burn Injury
}

\author{
Colleen N. Bartley, MD, Kenisha Atwell, MD, Bruce Cairns, MD and Anthony Charles, MD, MPH
}

\begin{abstract}
Racial and ethnic disparities in access to inpatient rehabilitation have been previously described for various injury groups; however, no studies have evaluated whether such disparities exist among burn patients. Their aim was to determine if racial disparities in discharge destination (inpatient rehabilitation, skilled nursing facility, home with home health, or home) following burn injury existed in this single-institution study. A retrospective analysis of all adult burn patients admitted to UNC Jaycee Burn Center from 2002 to 2012 was conducted. Patient characteristics included age, gender, burn mechanism, insurance status, percentage total body surface area (\%TBSA) burned, presence of inhalation injury, and hospital length of stay. Patients were categorized into one of three mutually exclusive racial or ethnic groups: White, Hispanic, or Black. Propensity score weighting followed by ordered logistic regression was performed in the analytical sample and in a subgroup analysis of patients with severe burns (TBSA > 20\%). For analysis, 4198 patients were included: 2661 White, 340 Hispanic, and 1197 Black. Propensity weighting resulted in covariate balance among racial groups. Black patients (OR: 1.58, 95\% CI: $1.23-2.03 ; P<.001$ ) were more likely than Whites to be discharged to a higher level of rehabilitation, whereas Hispanics were less likely (OR: $0.78,95 \% \mathrm{CI}: 0.38-1.58 ; P=.448$ ). In their subgroup analysis, Black (OR: 1.88, 95\% CI: 1.07-3.28; $P=.026)$ and Hispanic (OR: 1.53, 95\% CI: 0.31-7.51; $P=.603$ ) patients were more likely to discharge to a higher level of rehabilitation than White patients. Racial and ethnic disparities in discharge destination to a higher level of rehabilitative services among burn-injured patients exist particularly for Hispanic patients but not for Black or White burn patient groups. Further studies are needed to elucidate the potential sources of these disparities specifically for Hispanic patients.
\end{abstract}

There are approximately 40,000 burn injury-related hospitalizations in the United States annually. Due to improved clinical, operative, and critical care management of burn injury, specifically, early excision and grafting, burn injury survival rates as high as 96.8 percent have been achieved in the United States and other high income countries. ${ }^{1,2}$ With the well-documented decrease in burn mortality, morbidity is still significant and must be addressed in the postacute care setting, therefore determining the appropriate level of postinjury rehabilitation becomes paramount to full recovery and return to the highest functional status that is physically achievable.

Disparities in postinjury survival, morbidity, and functional outcomes have been reported among racial and ethnic minorities in the United States. ${ }^{3-6}$ Inpatient rehabilitation (IR), which is regarded as the highest level of rehabilitative services offered, can result in better functional outcomes and improve independent living after injury. ${ }^{7-9}$ There are studies documenting racial disparities in access to rehabilitation services, ${ }^{10,11}$ however, there is a paucity of data in the burn-injured patients. Discharge destinations occur along a

\footnotetext{
Department of Surgery, University of North Carolina at Chapel Hill, North Carolina Jaycee Burn Center, Chapel Hill, North Carolina

Address correspondence to Anthony Charles, MD, MPH, FACS, UNC School of Medicine, 4008 Burnett Womack Building, CB 7228, Chapel Hill, NC 27599-7228.Email:anthchar@med.unc.edu
}

spectrum, with IR being the highest level of rehabilitation, followed by skilled nursing facility (SNF), then home with home health aide assistance $(\mathrm{HH})$, and lastly home representing the lowest level of rehabilitation. Outside of acute inpatient rehabilitative services, few studies have evaluated other possible discharge destinations.

Patients with severe burns (TBSA > 20\%) who survive their injuries likely require higher levels of rehabilitation. Little is understood regarding the influence of race and ethnicity on discharge disposition for these patients. The aim of our study is to determine if racial or ethnic disparities exist in the likelihood of discharge to a higher level of rehabilitation following burn injury.

\section{METHODS}

We retrospectively analyzed the burn registry data collected from patients admitted to the University of North Carolina (UNC) Jaycee Burn Center from January 2002 to December 2012. The UNC Jaycee Burn Center is a 36-bed facility that averages over 1200 acute admissions annually and has been verified by the American Burn Association for pediatric and adult care. The UNC Institutional Review Board approved this study.

Patients 18 years of age and older who survived to discharge were included for analysis. Patients were excluded if they had undocumented discharge destination, were discharged to another acute care hospital or hospice, or if they left against medical advice. Additionally, patients were excluded in whom data were missing on race, sex, insurance status, type of burn 
injury, percentage total body surface area (\%TBSA) burn, intubation status, hospital length of stay (LOS), or intensive care unit (ICU) LOS.

Medical records of subjects identified by the UNC Burn database query were reviewed to verify baseline demographic data and injury characteristics. Injury characteristics of interest included burn etiology, \%TBSA burn, presence of inhalation injury, and use of mechanical ventilation during admission. Inhalation injury diagnosis was based on history, physical examination, and/or bronchoscopic examination. The primary exposure variable was race or ethnicity, operationalized as one of three mutually exclusive categories: White, Hispanic, or Black.

The outcome of interest in this study was discharge destination, categorized into one of four mutually exclusive but ordered discharge categories: home, home with home health services $(\mathrm{HH}), \mathrm{SNF}$, and IR. These categories represent increasing levels of care at discharge and, to some degree, increasing potential intensity of rehabilitation services available to an individual.

\section{Data Analysis}

Baseline patient and injury characteristics among White, Hispanic, and Black groups were compared using the Pearson chi-square test or Fisher's exact test for categorical variables and ANOVA for continuous variables in unadjusted cohorts. Firstly, the data analysis was performed comparing patient characteristics and variables based on racial or ethnic groups. We also calculated the standardized mean differences (SMD) to compare covariate imbalance before and after adjustment was made using propensity score weighting.

A multiassignment propensity score, estimated via a multinomial logistic regression, was used to derive the conditional probability of assignment according to race or ethnic category given the observed prehospital variables. Inverse probability of treatment assignment weighting (IPTW) was performed to achieve balance among race or ethnicity categories for selected prehospital variables. The prehospital variables included age, sex, insurance status, etiology, and \%TBSA; after literature review, our confounding variables for inclusion in the IPTW were decided a priori because these are associated with the outcome (discharge destination) and exposure (race or ethnicity). The SMD was compared between the unweighted and IPTW groups to assess balance. Following IPTW and balance assessment, ordered logistic regression was used to estimate odds ratios for discharge to a higher level of rehabilitation, with IR being the highest and home being the lowest level of rehabilitation. The regression was adjusted for in-hospital covariates (hospital LOS, ICU LOS, and mechanical ventilation) that were found to be associated with the outcome.

A subgroup analysis was completed in patients with TBSA $>20 \%$ using the same statistical methods. All statistical analysis was performed using Stata/MIP (version 15; Statacorp, College Station, TX). A $P$ value $<0.05$ was chosen to indicate statistical significance.

\section{RESULTS}

A total of 7641 patients were admitted during the study period (Figure 1) and 2310 patients < 18 years of age were excluded. Five hundred twenty-four patients were further excluded if not discharged to one of the four destinations of interest (home, home with home health, SNF, and IR) or no discharge destination was recorded. An additional 609 patients were excluded due to missing racial and ethnic demographic and clinical information. The remaining 4198 patients comprised of our study population.

\section{Patient Characteristics}

The mean age of the study population was $42.2 \pm 15.7$ years, with a majority being White $(63.4 \%)$, male $(73.5 \%)$, having experienced flame injury (53.6\%), and discharged home (90.3\%; Table 1). Black patients were significantly older than White or Hispanic patients $(44.2 \pm 15.5$ vs $42.3 \pm 16.0$ vs $33.6 \pm 10.9$ years, respectively; $P<.001)$. The majority of White $(72.5 \%)$ and Black (64.0\%) patients had insurance, whereas only $44.7 \%$ of Hispanic patients were insured. For each discharge destination, White patients (home $64.2 \%$, HH 54.4\%, SNF $57.7 \%$, and IR 58.2\%) were significantly more likely to be discharged to a higher level of rehabilitation than Hispanic patients (home 8.6\%, $\mathrm{HH} 4.6 \%$, SNF 0\%, and IR 1.5\%) and Black patients (home 27.2\%, $\mathrm{HH}$ $41.0 \%$, SNF $42.3 \%$, and IR 40.3\%).

\section{Propensity Matching}

Following IPTW for age, sex, insurance status, TBSA, type of burn, presence of inhalation injury, and intubation status, the SMD was calculated and balance was achieved. An ordered logistic regression was performed on the IPTW-adjusted sample. Black patients (OR: 1.58, 95\% CI: 1.23-2.03) were

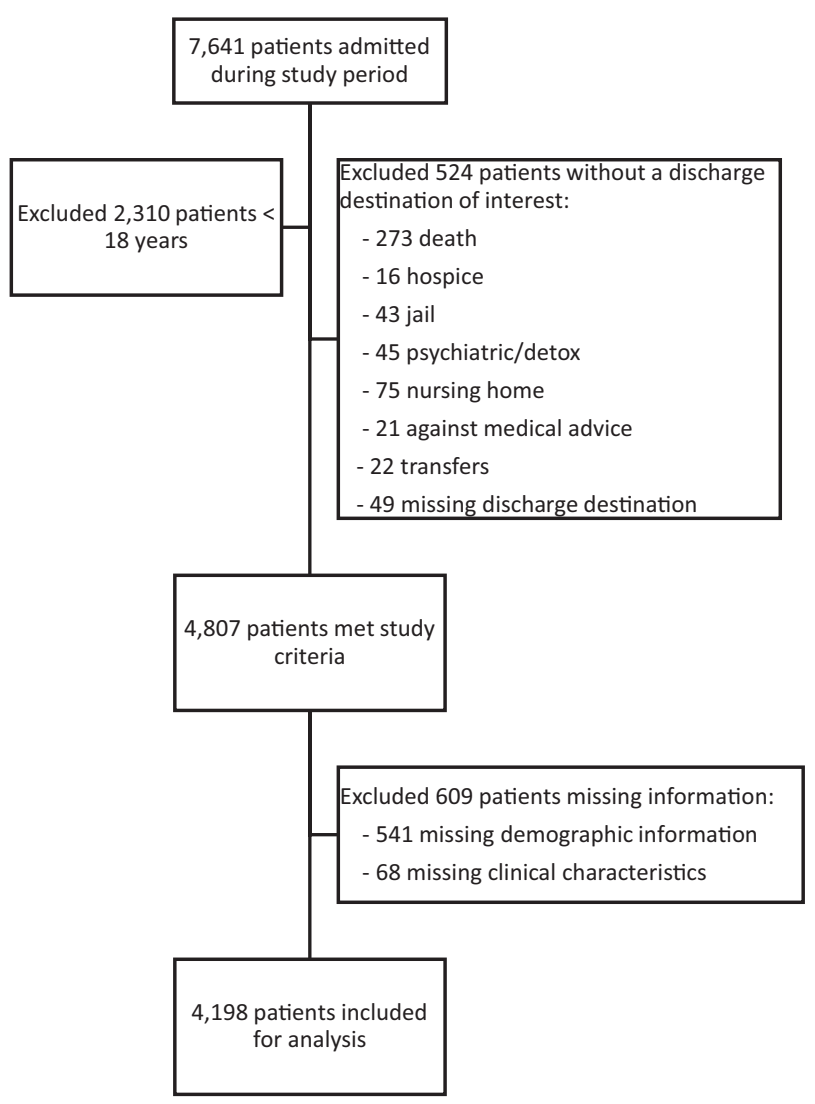

Figure 1. Flow diagram indicating inclusion and exclusion criteria. 
Table 1. Prehospital and clinical characteristics of burn injured patients by race

\begin{tabular}{|c|c|c|c|c|c|}
\hline Characteristics & Overall & White & Hispanic & Black & $P$ \\
\hline No. of patients & 4198 & 2661 & 340 & 1197 & \\
\hline Age $(\mathrm{yr})$, mean $\pm S D$ & $42.2 \pm 15.7$ & $42.3 \pm 16.0$ & $33.6 \pm 10.9$ & $44.2 \pm 15.5$ & $<.001$ \\
\hline \multicolumn{6}{|l|}{ Gender } \\
\hline M & $3084(73.5)$ & $2018(65.4)$ & $253(8.2)$ & $813(26.4)$ & \\
\hline $\mathrm{F}$ & $1114(26.5)$ & $643(57.7)$ & $8(7.8)$ & $384(34.4)$ & $<.001$ \\
\hline Have insurance & $2846(67.8)$ & $1928(72.5)$ & $152(44.7)$ & $766(64.0)$ & $<.001$ \\
\hline \multicolumn{6}{|l|}{ Type of burn } \\
\hline Flame & $2251(53.6)$ & 1596 & $145(6.4)$ & $510(22.7)$ & \\
\hline \multirow[t]{2}{*}{ Scald } & & $(70.9)$ & $159(9.6)$ & & \\
\hline & $1652(39.4)$ & $860(52.1)$ & & $633(38.3)$ & \\
\hline Other & $295(7.0)$ & $205(69.5)$ & $36(12.2)$ & $54(18.3)$ & $<.001$ \\
\hline \%TBSA, median (IQR) & $4.4(2-10)$ & $4(2-10)$ & $4.5(2-9.5)$ & $5(2-10)$ & .393 \\
\hline \multicolumn{6}{|l|}{ Discharge destination } \\
\hline Home & $3792(90.3)$ & 2435 & $327(8.6)$ & 1030 & \\
\hline $\mathrm{HH}$ & & $(64.2)$ & $12(4.6)$ & $(27.2)$ & \\
\hline SNF & $261(6.2)$ & $142(54.4)$ & $0(0.0)$ & $107(41.0)$ & \\
\hline \multirow[t]{3}{*}{ IR } & $78(1.9)$ & & $1(1.5)$ & & \\
\hline & & $45(57.7)$ & & $33(42.3)$ & \\
\hline & $67(1.6)$ & $39(58.2)$ & & $27(40.3)$ & $<.001$ \\
\hline $\begin{array}{l}\text { Hospital LOS (days) } \\
\text { mean } \pm S D\end{array}$ & $13.8 \pm 25.2$ & $12.7 \pm 25.2$ & $13.1 \pm 20.5$ & $16.5 \pm 26.2$ & $<.001$ \\
\hline ICU LOS (days), mean $\pm S D$ & $5.0 \pm 18.2$ & $4.6 \pm 17.9$ & $3.9 \pm 12.3$ & $6.1 \pm 20.0$ & .042 \\
\hline Inhalation injury & $318(7.6)$ & $199(32.58)$ & $25(7.86)$ & $94(29.56)$ & .909 \\
\hline Mechanical ventilation & $625(14.9)$ & $405(64.08)$ & $40(6.40)$ & $180(28.80)$ & .238 \\
\hline
\end{tabular}

$H H$, home with home health care; $S N F$, skilled nursing facility; $I R$, inpatient rehab; $I Q R$, interquartile range; $L O S$, length of stay; $I C U$, intensive care unit.

significantly more likely than Whites to be discharged to a higher level of rehabilitation (Table 2). Hispanics were less likely than Whites to be discharged to a higher level of rehabilitation, although this was not statistically significant (OR: $0.78,95 \%$ CI: $0.38-1.58 ; P=.448)$.

\section{TBSA > 20\%}

Unadjusted demographic data in our subgroup analysis for patients sustaining burns with TBSA > 20\% $(\mathrm{n}=371)$ are displayed in Table 3 . The average age was $40.6 \pm 14.6$ years, $60.7 \%$ were White, and $78.7 \%$ were male. Flame burn was the major type of burn (73.1\%) and median TBSA was 26\% (IQR: $22-36)$. The majority of patients were discharged home $(67.4 \%)$. There was no statistically significant difference in discharge destination $(P=.430)$. White patients were more likely to go home $(63.0 \%)$, go home with home health $(54.7 \%)$, and go to IR $(51.7 \%)$.

Following IPTW, balance was achieved in the covariates as described previously. Following ordered logistic regression, Blacks continued to be significantly more likely to discharge to a higher level of rehabilitation than Whites (OR: 1.88, 95\% CI: 1.07-3.28; $P=.026$; Table 4).

\section{DISCUSSION}

Disparities in access to care, treatment modalities, and outcomes in healthcare for various racial and ethnic groups have been documented across all levels of care in the United States. ${ }^{12,13}$ Usually, these differences are to the disadvantage of
Table 2. Ordered logistic regression estimating adjusted odds of discharge to a higher level of care

\begin{tabular}{lcr}
\hline Factor & Adjusted OR, 95\% CI & \multicolumn{1}{c}{$\boldsymbol{P}$} \\
\hline Race/ethnicity & & \\
$\quad$ White (ref) & $0.78(0.38-1.58)$ & .448 \\
Hispanic & $1.58(1.23-2.03)$ & $<.001$ \\
$\quad$ Black & $1.27(0.99-1.64)$ & .060 \\
Gender (male ref) & $1.05(1.04-1.06)$ & $<.001$ \\
Age (yr) & $3.48(2.44-4.98)$ & $<.001$ \\
Have insurance & & \\
Type of burn & & .886 \\
Flame (ref) & $1.02(0.79-1.31)$ & .006 \\
Scald & $1.90(1.20-3.01)$ & .001 \\
$\quad$ Other & $1.03(1.02-1.04)$ & .017 \\
Hospital LOS (days) & $0.98(0.97-1.00)$ & .248 \\
ICU LOS (days) & $1.32(0.82-2.12)$ & .033 \\
Inhalation injury & $1.51(1.03-2.20)$ & \\
Mechanical ventilation & & \\
\hline
\end{tabular}

Discharge to home is lowest level of care while discharge to inpatient rehab is highest level of care.

racial and ethnic minorities. This study is in contrast to most of the health care disparity literature. We demonstrate that Black patients are significantly more likely to be discharged to a higher level of rehabilitation than similarly matched White patients and in subgroup analysis of severely injured burn patients (TBSA > 20\%); this still held true.

Several studies exist demonstrating disparities in discharge destination following injury such as after traumatic brain 
Table 3. Prehospital and clinical characteristics of burn injured patients by race for patients with TBSA $>20 \%$

\begin{tabular}{|c|c|c|c|c|c|}
\hline Characteristics & Overall & White & Hispanic & Black & $P$ \\
\hline No. of patients & 371 & 225 & 26 & 120 & \\
\hline Age (years), mean $\pm S D$ & $40.6 \pm 14.6$ & $41.0 \pm 15.0$ & $32.6 \pm 11.6$ & $41.5 \pm 13.9$ & 0.014 \\
\hline Gender & & & & & 0.149 \\
\hline M & $292(78.7)$ & $181(62.0)$ & $23(7.9)$ & $88(30.1)$ & \\
\hline $\mathrm{F}$ & $79(21.3)$ & $44(55.7)$ & $3(3.8)$ & $32(40.5)$ & \\
\hline Have insurance & $266(71.7)$ & $167(74.2)$ & $11(42.3)$ & $88(73.3)$ & 0.003 \\
\hline \multicolumn{6}{|l|}{ Type of Burn } \\
\hline Flame & $271(73.1)$ & $171(63.1)$ & $20(7.4)$ & $80(29.5)$ & \\
\hline Scald & $69(18.6)$ & $31(44.9)$ & $4(5.8)$ & $34(49.3)$ & \\
\hline Other & $31(8.4)$ & $23(74.2)$ & $2(6.5)$ & $6(19.4)$ & 0.014 \\
\hline \%TBSA, median (IQR) & $26(22-36)$ & $26(22-35)$ & $28.5(23-42)$ & $26(22-37)$ & 0.686 \\
\hline \multicolumn{6}{|l|}{ Discharge destination } \\
\hline Home & $182(67.4)$ & $177(63.0)$ & $22(7.8)$ & $82(29.2)$ & \\
\hline $\mathrm{HH}$ & $44(16.3)$ & $29(54.7)$ & $3(5.7)$ & $21(39.6)$ & \\
\hline SNF & $28(10.4)$ & $4(50.0)$ & $0(0.0)$ & $4(50.0)$ & \\
\hline IR & $16(5.9)$ & $15(51.7)$ & $1(3.5)$ & $13(44.8)$ & 0.430 \\
\hline \multicolumn{6}{|l|}{ Hospital LOS (days), } \\
\hline mean $\pm S D$ & $53.7 \pm 58.7$ & $51.5 \pm 64.3$ & $53.0 \pm 37.1$ & $58.0 \pm 51.1$ & 0.619 \\
\hline ICU LOS (days), mean $\pm S D$ & $32.9 \pm 46.0$ & $31.3 \pm 48.6$ & $27.0 \pm 26.4$ & $37.0 \pm 44.2$ & 0.437 \\
\hline Inhalation injury & $97(26.2)$ & $50(51.6)$ & $8(8.3)$ & $39(40.2)$ & 0.101 \\
\hline Mechanical ventilation & $178(48.0)$ & $104(58.4)$ & $12(6.7)$ & $62(34.8)$ & 0.617 \\
\hline
\end{tabular}

$H H$, home with home health care; $S N F$, skilled nursing facility; $I R$, inpatient rehab; $I Q R$, interquartile range; $L O S$, length of stay; $I C U$, intensive care unit.

Table 4. Ordered logistic regression estimating the adjusted odds of discharge to a higher level of care depending on race/ethnicity for patients with TBSA > 20\%

\begin{tabular}{lcc}
\hline Factor & Adjusted OR, 95\% CI & $P$ \\
\hline Race/ethnicity & & \\
$\quad$ White (ref) & $1.53(0.31-7.51)$ & .603 \\
$\quad$ Hispanic & $1.88(1.07-3.28)$ & .026 \\
$\quad$ Black & $1.13(0.56-2.27)$ & .732 \\
Gender (male ref) & $1.04(1.02-1.06)$ & $<.001$ \\
Age (yr) & $4.61(2.18-9.72)$ & $<.001$ \\
Have insurance & & \\
Type of burn & & \\
$\quad$ Flame (ref) & $0.93(0.47-1.82)$ & .826 \\
Scald & $2.94(0.92-9.38)$ & .069 \\
$\quad$ Other & $1.01(1.00-1.02)$ & .191 \\
Hospital LOS (days) & $1.00(0.99-1.02)$ & .882 \\
ICU LOS (days) & $1.13(0.58-2.19)$ & .722 \\
Inhalation injury & $2.39(1.17-4.87)$ & .017 \\
Mechanical ventilation & & \\
\hline
\end{tabular}

injury (TBI). Meagher et al evaluated likelihood of discharge to a higher level of rehabilitation, taking into account the different levels of rehabilitation services. ${ }^{14}$ They determined that both Black and Hispanic patients were less likely to be discharged to a higher level of rehabilitation than White patients. Both Shafi et $\mathrm{al}^{15}$ and Englum et $\mathrm{al}^{10}$ demonstrated similar findings, though they did not account for the different discharge destinations. In another study, Bowman et al evaluated patients with moderate to severe TBI. Black and Hispanic patients were categorized together and similarly found to be significantly less likely to be discharged to rehabilitation services. ${ }^{15}$

Our findings demonstrate that Black patients do not always receive less rehabilitative care, particularly in the burn cohort; however, Hispanic patients compared with White and Black patients were more likely to be discharged to a lower level of rehabilitative care. More recently, Skolarus et al, using national Medicare data, looked at over 180,000 Black and White patients hospitalized with a primary diagnosis of stroke. They assessed the proportion of stroke survivors receiving physical, occupational, and speech and language therapy in each postacute care setting (IR facility, SNF, and home health agency), minutes of therapy, and number of transitions between settings. They showed that Black stroke patients were more likely to receive each type of therapy than White stroke patients. Compared with White stroke patients, Black stroke patients received more minutes of physical therapy, occupational therapy, and speech and language therapy. ${ }^{16}$

One possible explanation for our findings may be the increased proportion of insured Black patients at $64.0 \%$ and even when uninsured at admission, and Black patients are more likely to eligible for indigent care or Medicaid before discharge as U.S. citizens. Insurance status is an important predictor of hospital disposition and access to specialized posthospital care. ${ }^{17,18}$ Sacks et al evaluated likelihood of discharge to rehabilitation, skilled nursing facilities, and home health for a cohort of trauma patients. ${ }^{19}$ Uninsured patients were found to have the lowest odds of being discharged to rehabilitation, SNF, and $\mathrm{HH}$. However, we controlled for and propensity matched admission insurance status among racial and ethnic groups in our analysis. 
Hispanic patients in our study were less likely to be discharged to higher level of rehabilitation services overall, which is consistent with the literature. Furthermore, no Hispanic patients went to a SNF and few were discharged to IR. The small number of Hispanic patients could explain some of these nonsignificant differences. Additionally, Hispanic patients were the least insured group (44.7\%). They were, however, more likely to receive higher-level ser-vices in the severe burn subpopulation analysis. This finding suggests that severity of injury may be important in deter-mining if patient will receive higher level of care. Hispanics comprise nearly 17 percent of the U.S. population, and this proportion is anticipated to increase to 30 percent by $2060 .^{20}$ Hispanics are a diverse ethnic group that includes many dif-ferent cultures, races, and nationalities. Factors leading to our observed disparity include language, ${ }^{21}$ lack of insurance, illegal immigration status and fear of deportation, mistrust, and illiteracy. Furthermore, the sociocultural norms in the Hispanic population may also play a role in discharge destination. Family is very important in the Hispanic culture and the family participates in important medical decisions and indeed delivery of care at home.

Limitations of this study include those attributable any study with retrospective methodology. Patient support at home (ie, spouse, family member, and friend) can play a significant role in discharge destination, particularly for burn patients who require wound care. Given the retrospective approach of this study, however, this information was not collected and therefore not available for analysis. Furthermore, patient and family attitudes regarding care and where that care is performed also play a role in disposition decisions. Qualitative studies looking into communication between the medical team and families should be done to further elucidate this area. Furthermore, in any study on race and ethnicity, missing data or possible misclassification may occur, particularly if race is not self-assigned. Socioeconomic status (SES) is often interrelated to race and therefore health outcomes. Although we did include insurance status as a proxy for SES, other indicators such as median household income, occupation, and educational attainment were not included. Finally, it is well known that variations in burn care exist across the United States. As such, our single-institution findings may not be generalizable. Future research should be conducted to evaluate for racial and ethnic disparities in discharge destination after burn injury across the nation so that the medical community can better address disparities in medical care in the United States.

\section{CONCLUSIONS}

Racial and ethnic disparities in discharge destination to a higher level of rehabilitative services among burn-injured patients exist, particularly for Hispanic but not for Black or White burn patient groups. Further studies are needed to elucidate the potential sources of these disparities specifically for Hispanic patients. Every effort must be made to understand some of the sociocultural determinants of health and health literacy in the burn population.

\section{ACKNOWLEDGEMENT}

This work was supported by the National Institute of Health under award number 5T32GM008450-23.

\section{REFERENCES}

1. American Burn Association 2016 Fact Sheet; available from http://www ameriburn.org/who-we-are/media/burn-incidence-fact-sheet; accessed Feb. 162018.

2. Klein MB, Goverman J, Hayden DL et al.; Inflammation and Host Response to Injury, and Large-Scale Collaborative Research Program. Benchmarking outcomes in the critically injured burn patient. Ann Surg 2014;259:833-41.

3. Fauerbach JA, Engrav L, Kowalske K et al. Barriers to employment among working-aged patients with major burn injury. J Burn Care Rehabil 2001;22:26-34

4. Goverman J, Mathews K, Goldstein R et al. Adult contractures in burn injury: a burn model system national database study. J Burn Care Res 2017;38:e328-36.

5. Arango-Lasprilla JC, Rosenthal M, Deluca J, Cifu DX, Hanks R, Komaroff E. Functional outcomes from inpatient rehabilitation after traumatic brain injury: how do Hispanics fare? Arch Phys Med Rehabil 2007;88:11-8.

6. Arango-Lasprilla JC, Rosenthal M, Deluca J et al. Traumatic brain injury and functional outcomes: does minority status matter? Brain Inj 2007;21:701-8.

7. Greene NH, Pham TN, Esselman PC, Rivara FP. Variation in inpatient rehabilitation utilization after hospitalization for burn injury in the United States. J Burn Care Res 2015;36:613-8.

8. Sliwa JA, Heinemann A, Semik P. Inpatient rehabilitation following burn injury: patient demographics and functional outcomes. Arch Phys Med Rehabil 2005;86:1920-3.

9. Spires MC, Bowden ML, Ahrns KS, Wahl WL. Impact of an inpatient rehabilitation facility on functional outcome and length of stay of burn survivors. J Burn Care Rehabil 2005;26:532-8.

10. Englum BR, Villegas C, Bolorunduro $\mathrm{O}$ et al. Racial, ethnic, and insurance status disparities in use of posthospitalization care after trauma. J Am Coll Surg 2011;213:699-708.

11. Klein MB, Lezotte DC, Heltshe $S$ et al. Functional and psychosocial out comes of older adults after burn injury: results from a multicenter database of severe burn injury. J Burn Care Res 2011;32:66-78.

12. Nelson A. Unequal treatment: confronting racial and ethnic disparities in health care. J Natl Med Assoc 2002;94:666-8.

13. Shafi S, de la Plata CM, Diaz-Arrastia R, Bransky A, Frankel H, Elliott AC et al. Ethnic disparities exist in trauma care. J Trauma 2007;63:1138-42.

14. Meagher AD, Beadles CA, Doorey J, Charles AG. Racial and ethnic disparities in discharge to rehabilitation following traumatic brain injury. J Neurosurg 2015;122:595-601.

15. Bowman SM, Martin DP, Sharar SR, Zimmerman FJ. Racial disparities in outcomes of persons with moderate to severe traumatic brain injury. Med Care 2007;45:686-90.

16. Skolarus LE, Feng C, Burke JF. No racial difference in rehabilitation therapy across all post-acute care settings in the year following a stroke. Stroke 2017;48:3329-35.

17. Asemota AO, George BP, Cumpsty-Fowler CJ, Haider AH, Schneider EB. Race and insurance disparities in discharge to rehabilitation for patients with traumatic brain injury. J Neurotrauma 2013;30:2057-65.

18. Kane WG, Wright DA, Fu R, Carlson KF. Racial/ethnic and insurance status disparities in discharge to posthospitalization care for patients with traumatic brain injury. J Head Trauma Rehabil 2014;29:E10-7.

19. Sacks GD, Hill C, and Rogers SO, Jr. Insurance status and hospital discharge disposition after trauma: inequities in access to postacute care. J Trauma 2011;71:1011-5. doi:10.1097/TA.0b013e3182092c27

20. https://census.gov/content/dam/Census/library/publications/2015/demo/p25-1143.pdf; accessed 2 Mar 2018.

21. Betancourt JR, Green AR, Carrillo JE, Ananeh-Firempong O, 2nd. Defining cultural competence: a practical framework for addressing racial/ethnic disparities in health and health care. Public Health Rep 2003;118:293-302. 